\title{
Design of a Simple High-Altitude Rescue Buffer Device
}

\author{
Shan Chen ${ }^{\star}$, Junchun Ma and Baoming Qiang \\ Xi'an Research Institute of High Technology, Xi'an, Shanxi, China, 710025 \\ *Corresponding author
}

Keywords: Buffer device, High-altitude, Rescue.

\begin{abstract}
With the increasing number of high-rise buildings in cities, people have more and more chances to encounter dangers in high-rise buildings. How to escape safely and quickly has become a hot research issue. Based on this, this paper designs a hydraulic damped high-altitude rescue buffer device based on the principle of hydraulic transmission. On the premise of fully understanding the current market situation of the retarder, the design scheme of hydraulic damper type is put forward at first. Then, according to the five components of the hydraulic system, the overlap of the overall structure of the system is completed. Finally, the model is developed by SolidWorks, a three-dimensional aided design software, and the engineering drawings of the whole system components are designed.
\end{abstract}

\section{Introduction}

With the continuous development of science and technology in China and the continuous improvement of manufacturing industry capacity, the safety of high-rise buildings has been paid more and more attention. From the slower market, there are mainly the following types: Drum type retarder, screw type retarder, friction damper type retarder and hydraulic damper type retarder.

From the current market, there are various kinds of retarders. At the same time, people are looking forward to the emergence of retarders which are easy to operate and can control the speed of descent by themselves. That is to say, the new type of retarder should embody more humanistic design ideas, take into account the factors of different weights, so that the trapped personnel can use it without complicated training, that is, it needs to have simple structure, easy installation, short setup time, fast evacuation personnel and so on.

\section{Overall Scheme Design}

The design of hydraulic damper retarder is mainly aimed at the situation that people are trapped and unable to use elevators, stairs or lifesaving passages normally when fire or danger occurs in high-rise buildings. Therefore, on the premise of safety, the design should make the structure of the device as simple as possible, the volume as small as possible, and the users can flee the dangerous zone quickly. The hydraulic damped retarder is composed of a hydraulic cylinder and a throttle valve. Its working principle is shown in Fig. 1 below.

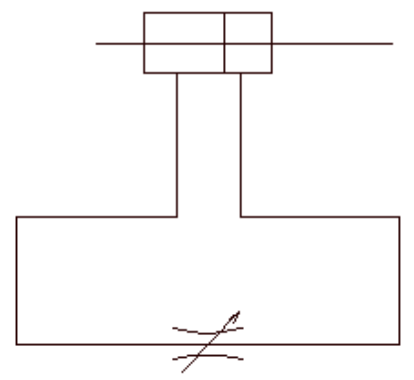

Figure 1. Schematic diagram of hydraulic damper retarder 
The specific working process is as follows: The trapped people wearing safety life jackets fell because of their self-esteem. The wire rope connected to the human body then drives the drum to rotate, and the drum drives the crank-connecting rod mechanism at both ends to rotate. The function of crank-connecting rod mechanism is to convert the circular motion of the drum into the linear reciprocating motion of the double-acting hydraulic cylinder with double piston rods, and complete the conversion of motion. Because the crank connecting rod mechanism is connected with the hydraulic cylinder rod, the hydraulic cylinder, under the action of the crank, presses the hydraulic oil from one oil chamber to another. In order to control the descending speed of personnel, the flow of oil must be controlled by throttling device. By controlling the throttle orifice size of throttle valve, controlling the oil flow speed, and then through the internal feedback of the system, controlling the rotational speed of the drum, the purpose of controlling the descending speed of the personnel is achieved.

\section{Structural Design and Component Selection}

Hydraulic transmission system is generally composed of five parts: energy device, actuator, control throttle device, auxiliary device and transmission medium. In the specific design process, according to these five parts, the components of each part are selected, calculated and finalized in turn, and then the overall structure and function of the device are finalized.

\section{Selection of Energy Devices}

In the hydraulic transmission system, engineers usually use hydraulic pump as the energy device. Its advantages are obvious, such as smooth transmission, large load, step less speed regulation, etc. Its disadvantages also follow, such as complex structure, too large volume, heavy weight, lack of flexibility, inconvenient device movement, etc. Therefore, this paper adopts passive hydraulic system, does not use any mechanism driven by external power of energy as the energy device, and directly connects the two oil chambers of hydraulic cylinder through oil pipes, thus avoiding the heavy design problem of the device in principle.

\section{Selection of the Actuator}

In the hydraulic transmission device, the main actuators are hydraulic cylinder and hydraulic motor, whose main function is to convert hydraulic energy into mechanical energy. For the hydraulic damped retarder in this paper, its actuator is hoisting mechanism and hydraulic cylinder. First, the selection of hoisting mechanism. When high-level rescue of personnel or materials is carried out, the movement of rescued personnel and materials is in the vertical direction. Therefore, the hoisting mechanism is selected as the intermediate device, and the movement of the personnel in the vertical direction is converted into the circular motion of the drum through the drum, which drives the rotation of the drum and greatly extends the height of rescue. Secondly, the selection of hydraulic cylinder, because the hoist mechanism converts the movement of personnel into circular movement, at the same time considering that the passive system is composed of hydraulic cylinder and various auxiliary mechanisms. Therefore, as long as the circular motion of the drum is converted into the linear motion of the hydraulic rod, which drives the hydraulic rod to move in the linear direction, the mechanical motion conversion between the actuators can be realized, thus connecting the extension and retraction speeds of the hydraulic rod with the rising and falling speeds of personnel.

\section{Selection of Control Throttle Device}

In a hydraulic transmission system, no matter how complex its functions and the number of components are, its oil circuit is composed of hydraulic components that control the direction, pressure and flow of oil in the hydraulic system. From the point of view of hydraulic damper, the function of the control device is to control the descending speed of the personnel. Belt brake and throttle valve are the ways to control speed. Finally, the control element--throttle valve is adopted in this paper. The following aspects are mainly considered: First of all, the belt brake is adopted, and the 
key point of the whole system falls on the friction torque between the belt and the drum. To control the uniform speed, deceleration or stop of the personnel in the descending process, it is necessary to control the torque applicator, which will add additional control mechanisms and complicate the system structure. Moreover, the emergency rescue situation is full of uncertain factors, which will affect the application of friction torque in rainy days or humid environments, causing extremely easy slippage and limiting the time and scope of use. Secondly, from the cost point of view, the cost price of a single throttle valve is 30 to $40 R M B$. Compared with the cost price of belt brake thousands $R M B$, its manufacturing cost is low, which is convenient for large-scale promotion.

\section{Selection of Auxiliary Devices}

Bracket: It is the basic frame and main bearing mechanism of the whole device. In order to ensure that the system can work steadily throughout the normal working process, the triangular structure is adopted in the design of the bracket. Crankshaft: It is a force transmission mechanism, which connects the hydraulic cylinder and the drum, and converts the circular motion of the drum into the linear motion of the hydraulic cylinder. Tee: It is the interface of the whole passive hydraulic system to replenish the oil. When it is necessary to replenish the oil to the system, the third interface is used to replenish the oil to the oil chambers on both sides of the hydraulic cylinder. When working normally, the third oil outlet is plugged with a plug to form a closed space and form a passive hydraulic transmission system.

\section{Selection of Transmission Medium}

Commonly used hydraulic oils are classified according to working pressure and viscosity: L-HL hydraulic oil, L-HM anti-wear hydraulic oil, L-HV low temperature anti-wear hydraulic oil, L-HS Low setting anti-wear hydraulic oil, L-HG hydraulic guide oil, fire-resistant hydraulic oil. Viscosity grades of hydraulic oils are divided into 22, 32, 46, 68, 100, etc. at $40 \mathrm{C}$, but their properties have their own characteristics. Because the hydraulic cylinder is used as the actuator, according to the relevant regulations of the state, the oil in the whole hydraulic circuit must be made of 46 at the same time, $\mathrm{L}-\mathrm{HL}$ oil is selected because the working pressure of the system is less than $7 \mathrm{MPa}$.

\section{Usage Method}

Fix the Descent control device on the balcony, ceiling, corridor and other places. When there is a fire, the evacuees should fasten their safety belts and then fasten the safety belts to the rope buckles of Descent control device. By adjusting the throttle opening to different positions, the oil flow through the throttle valve is different in unit time. According to the requirements, this Descent control device has designed three different downshifts and one emergency stop gear. The first gear is set within 50 $\mathrm{Kg}$, the second gear is set within $50 \mathrm{~kg}$ to $70 \mathrm{Kg}$, the third gear is set above $70 \mathrm{~kg}$, and the fourth gear is set for emergency stop.

\section{Modeling and Simulation}

In this paper, the process of modeling needs to be continuously improved. First, the development of the model. SolidWorks is used to model the hydraulic damping Descent control device. As the actual size of Descent control device is completely uncertain before the product is completely finalized, the previous model development mainly verifies the feasibility of the scheme in terms of scheme, principle and structure. Secondly, the determination of component model. On the basis of the feasibility of the scheme, relevant calculations are carried out. According to specific national standards, the parameters of hydraulic components are integrated into standard parts as far as possible to reduce the cost of the whole system. Finally, the parameters are improved. The component size and installation size of the model initially developed in the early stage are improved, and the calculated parameters are kept consistent with the three-dimensional model as far as possible. Due to the limitation of space, the modeling process of hydraulic cylinder is introduced as an example. 
Previously, through market research, the model of hydraulic cylinder has been determined MOD30 *200-50, and the parameters of hydraulic cylinder have been checked and calculated. The components of each module have been clearly understood. According to the analysis idea, firstly, using the top-down analysis idea, according to the mastered principle, structure and composition, the hydraulic cylinder is divided into: rear end cover, cylinder barrel, piston, piston rod, front end cover, fastening connecting rod and sealing device.

\section{Piston}

The diameter of the piston is $30 \mathrm{~mm}$ and the diameter of the piston rod is $16 \mathrm{~mm}$, therefore, the piston and the piston rod are connected by threads. The entire piston thickness is $10 \mathrm{~mm}$. The normal operation of the hydraulic cylinder depends on the sealing effect between the piston and the cylinder barrel to isolate the oil inlet chamber and the oil outlet chamber from each other. According to the needs, the sealing ring for the hole is selected, and OSI type piston special seal or UPI type piston and piston rod can be used.

\section{Piston Rod}

The two-way hydraulic cylinder is selected in the form of cylinder body fixation. The one-way stroke of the piston rod is $200 \mathrm{~mm}$, the total length of the piston rod is $400 \mathrm{~mm}$, and the total stroke of the hydraulic cylinder is $600 \mathrm{~mm}$. In order to avoid oil leakage at the end of the hydraulic cylinder when the piston rod works, a sealing ring should be used for the shaft between the piston rod and the end cover. The sealing ring should be used for IDI type piston rod or UPI type piston and piston rod to strengthen the sealing performance of the device. When installing, pay attention to whether the hydraulic cylinder will touch the ground. As the hydraulic cylinder works unilaterally, an adjustable section of $50 \mathrm{~mm}$ is left at one end, which is convenient for installation and debugging of the hydraulic cylinder. The model is shown in fig. 2.

\section{Front and Rear End Caps}

Front and rear end caps are fixed mechanisms of the whole device, which fix cylinder, piston rod and other parts together as a whole. At the same time, for the double-acting rod, front and rear end caps also play a guiding role, providing the basic direction of motion for the piston rod. The model is as follows Fig. 3 Show.

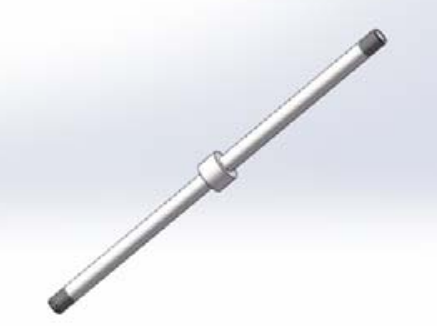

Figure 2. Piston rod model
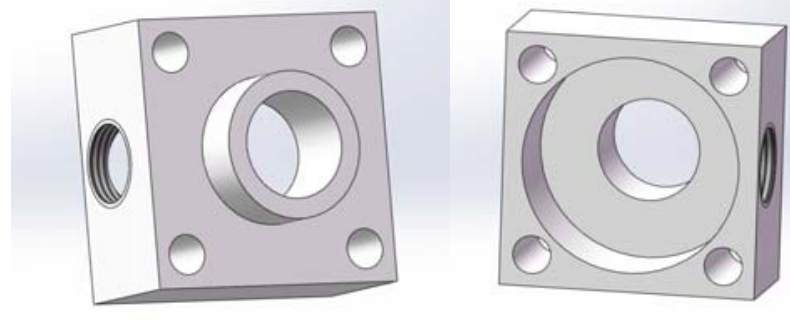

Figure 3. Model of end cap

(1) Drawing command, select up-looking datum plane, make piston diameter $30 \mathrm{~mm}$, set end cap length $40 \mathrm{~mm}$, width $40 \mathrm{~mm}$, height $20 \mathrm{~mm}$ Rectangular block;

(2) For stretching command, the front guide orifice is a cylindrical guide orifice with an outer diameter of $22 \mathrm{~mm}$ and a height of $6 \mathrm{~mm}$.

(3) For the first stretching excision, a cylinder with a diameter of $32 \mathrm{~mm}$ and a depth of $15 \mathrm{~mm}$ was excised by stretching.

(4) The second stretching excision, in front of the base plane, stretching excision diameter of 18 mm through-hole cylinder;

(5) Circumferential array, making four through holes with diameter of $8 \mathrm{~mm}$, center distance of 30 $\mathrm{mm}$, fastening the installation position of connecting rod;

(6) Choose G1/4 threaded hole through hole, $8 \mathrm{~mm}$ from the base of end cap, which is the import and export of oil. 


\section{Cylinder}

The piston diameter of the hydraulic cylinder is $30 \mathrm{~mm}$. The cylinder with inner diameter $30 \mathrm{~mm}$, outer diameter $32 \mathrm{~mm}$ and wall thickness $2 \mathrm{~mm}$ is selected. The oil inlet and outlet at both ends are set as G1/4 threaded holes, and the center distance between the two holes is $186 \mathrm{~mm}$. The model is as follows Fig.4 shows.

\section{Fastening Connecting Rod}

The fastening connecting rod is a double-head bolt, which is easy to draw. You can either draw it by yourself or look for it in the standard parts library on Midi's secondary development platform. The design of double-headed bolt has been parameterized. You can choose the corresponding parameters. Here you can choose the thread M8. Model As shown in Fig.5.

\section{Improvement of the Model}

The hydraulic cylinder shall be installed from inside to outside and from left to right, and parts shall be assembled as far as possible according to its working position and structural distribution. It is mainly to add the constraint relation between each component, and the added constraint shall conform to the actual working conditions. After the installation is completed, the model shall be functionally checked. Firstly, the model is checked for coherence to detect whether there are too many constraints and unreasonable constraints in the assembly process and whether the assembled parts coincide with each other. Secondly, the motion simulation of the model is aimed at the physical simulation of the hydraulic components in the working process to observe whether each component can work normally under the given conditions. The model is shown in Fig.6 below.

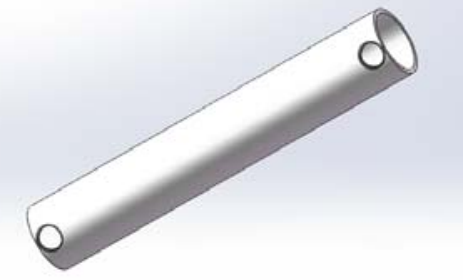

Figure 4. Cylinder model

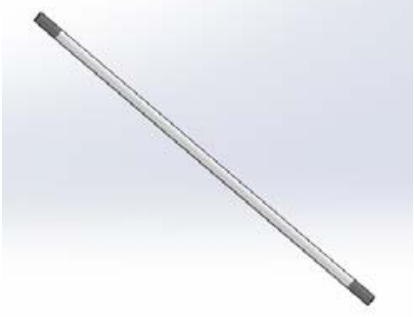

Figure 5. Fastener model

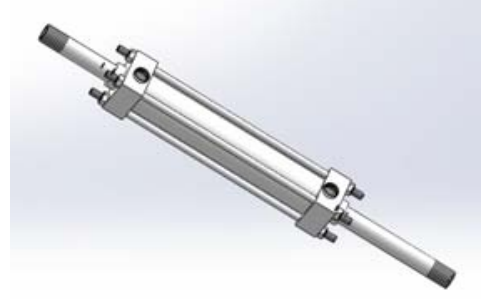

Figure 6. Hydraulic cylinder model

The above is the process of using SolidWorks software to model the hydraulic cylinder as an example. The other component models are similar to this process, and finally the device is obtained. The model is shown in Fig.7.

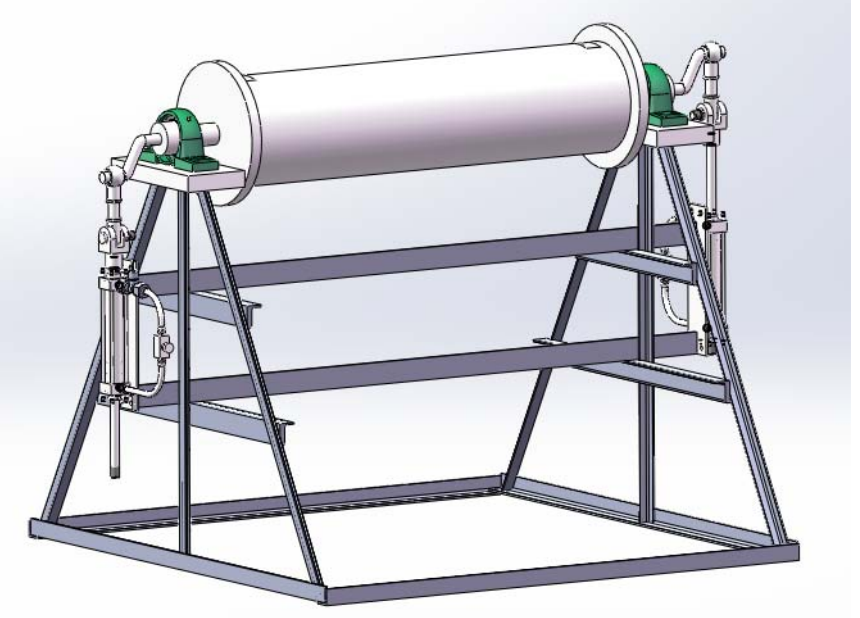

Figure 7. Hydraulic damper model 


\section{Conclusions}

Buffer and rescue devices in high-rise buildings are a direction of the development of safety products in public places, and they have a large market in today's high-rise buildings. In this paper, the design of the device is mainly based on the principle of hydraulic damper, and the main work is as follows: (1) Conception of the scheme. By collecting the products of the retarder on the market at present, the design scheme of hydraulic damper is put forward. (2) Overlapping of the overall structure of the system. According to the five components of the hydraulic system, the components of the system are determined one by one. (3) Selection of components. According to the requirement, the parameters of device components are selected. (4) Model development. The model is developed by SolidWorks, a three-dimensional aided design software, and the related dimensions are perfected. The engineering drawings of the whole system components are designed.

\section{Reference}

[1] Han Yuyong, Liu Yunhui and Li Ziguo. Design of Life Saving Descent control device Based on Dynamic Theory Analysis and Simulation[J]. Fire Science and Technology, 2015, (10): 1380-1386.

[2] Wu Shuqin, Suo Shuangfu, Huang Tao, Zheng Wenjiao, Liu Xiangfeng and Wang Yuming. Research on Energy Dissipation of Self-cooling Friction Descent control device [J]. Mechanical Design, 2013, (03): 86-88+92.

[3] Wang Wenkai, Song Haichao. Design of a Speed Regulation Braking System for a Multifunctional Escape Descent control device [J]. Hydraulic and Pneumatic, 2012, (11): 35-37.

[4] Wei Jianjun. Research and Development of Centrifugal Friction High-rise Escape Descent control device [J]. Manufacturing Automation, 2012, (04): 90-92.

[5] Lan Zimian, Zhong Qing. A Design of Escape Hydraulic Descent control device [J]. Hydraulic and Pneumatic, 2011, (12): 7-8. 